DOI: https://doi.org/10.14311/TPFM.2022.008

\title{
INVESTIGATION OF TRANSONIC FLOW THROUGH LINEAR CASCADE WITH SINGLE BLADE INCIDENCE ANGLE OFFSET
}

\author{
Jiř́ Fürst ${ }^{1}$, Josef Musil ${ }^{1,2}$, David Šimurda ${ }^{2}$ \\ ${ }^{1}$ Faculty of Mechanical Engineering, Czech Technical University in Prague, Karlovo Náměstí \\ 13, 12135 Prague 2, Czech Republic. \\ 2 Institute of Thermomechanics, Czech Academy of Sciences, Dolejškova 5, 18200 Prague 8, \\ Czech Republic.
}

\begin{abstract}
The contribution deals with numerical and experimental investigation of the effect of incidence angle offset in a two-dimensional section of a flat linear blade cascade in a high-speed wind tunnel. The aim of the work is to complement ongoing research of quasi-stationary approximation of aerodynamic flutter by examination of setups leading to transonic flow regimes. The numerical simulations were realized by finite-volume, in-house code developed on top of the open-source software package OpenFOAM. The experiments were conducted in correspondence with the setting of numerical simulations. The comparison of experimental and numerical data is presented on the isentropic Mach number distributions at various locations in the blade cascade. The description of transonic flow structures in the vicinity of blades is also provided.
\end{abstract}

Keywords: compressor cascade, flutter, CFD, finite volumes, experimental validation, transonic.

\section{Introduction}

The presence of a blade flutter; a phenomenon of an unstable and self-excited vibration motion of a blade in an airstream induced by the interaction between fluid and the blade, in large-output turbomachines can cause critical blade deformations eventually leading to fatigue cracks and consequently destruction of the operating machine. Whereas the prediction of flutter boundaries retains a fundamental relevance in industrial blade design due to the ever-increasing demand on performance and efficiency, comprehensible solution resolving all the relevant aspects of the flutter has not been yet provided. The major unknown in the blade flutter prediction is the aerodynamic unsteady pressure loading generating blade vibrations. The experimental assessment of the pressure loading in real machines is prohibitively expensive and technologically complicated so only few test facilities dedicated to flutter research exist worldwide, e.g. [1-4].

In the following, comparison of experimental measurements and numerical computations of simplified, quasi-stationary approach of the the blade flutter approximation will be provided. The contribution is a follow-up to the ongoing research [5], [6] of blade flutter investigation where the assumption of that the dynamic component of the overall blade loading is considerably smaller than the level of the overall static loading component is adopted in order to substitute timedependent blade loadings by a series of static loadings for a range of blade incidence angle offsets. The experimental part of the research was carried on the new setup for controlled flutter testing operating at the Institute of Thermomechanics of the Czech Academy of Sciences [7]. Numerical computations were realized by finite-volume, in-house code developed on the top of open-source software package OpenFOAM. For the first phase of the research, five simple flat blades in linear two-dimensional cascade with prescribed harmonic torsional movement of the middle blade was used.

In the previous study [6], the subsonic flow regimes were examined. The present contribution is focused on investigation of transonic flow in the cascade and description of subsequent flow structures in the cascade. Static pressure readings were measured in various positions in the cascade in the experimental part and then used for evaluation of the isentropic Mach number values compared with $\mathrm{CFD}$ results. 


\section{Experimental and Computational Setup}

\subsection{Formulation of the problem}

Geometrical configuration of the cascade is the same as in [6]. The cascade consists of five flat profiles positioned in the middle of the straight channel; see Figure 1. Blades are enumerated from the channel floor up. The middle blade (blade no. 3) is located in the center of the channel and has a variable incidence angle $\alpha$. The blade profile is a flat plate of a chord of $c=120 \mathrm{~mm}$ and a thickness of $t=5 \mathrm{~mm}$. The leading and the trailing edges are circular. The height and length of the computational domain is $h=195 \mathrm{~mm}$ and $w=1000 \mathrm{~mm}$, respectively. The setting angle of blades in the cascade is $\beta=31.5^{\circ}$ and the pitch is $s=74.52 \mathrm{~mm}$. The air enters the channel from the left upstream of the cascade.

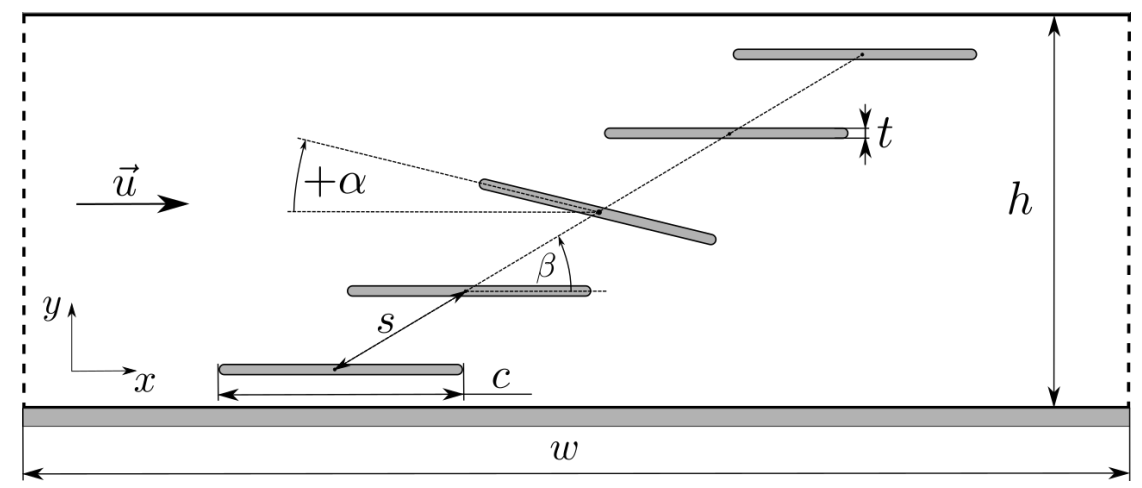

Figure 1: Geometry of the cascade.

The flow regime is characterized by a reference Mach number $M_{o}$ evaluated from 20 wall pressure readings $p_{0, k}(k=1, \cdots, 20)$ and evenly distributed along a line parallel to the cascade at distance $d=12 \mathrm{~mm}$. Probe separation downstream of the cascade is $14.9 \mathrm{~mm}$ and the first probe is located at the lower left end of the line. The reference outlet pressure is then calculated as $p_{0, a v g}=\frac{1}{20} \sum_{k=1}^{20} p_{0, k}$ and the reference Mach number is

$$
M_{o}=\sqrt{\frac{2}{\gamma-1}\left[\left(p_{0, a v g} / p_{1, t o t}\right)^{\frac{1-\gamma}{\gamma}}-1\right]},
$$

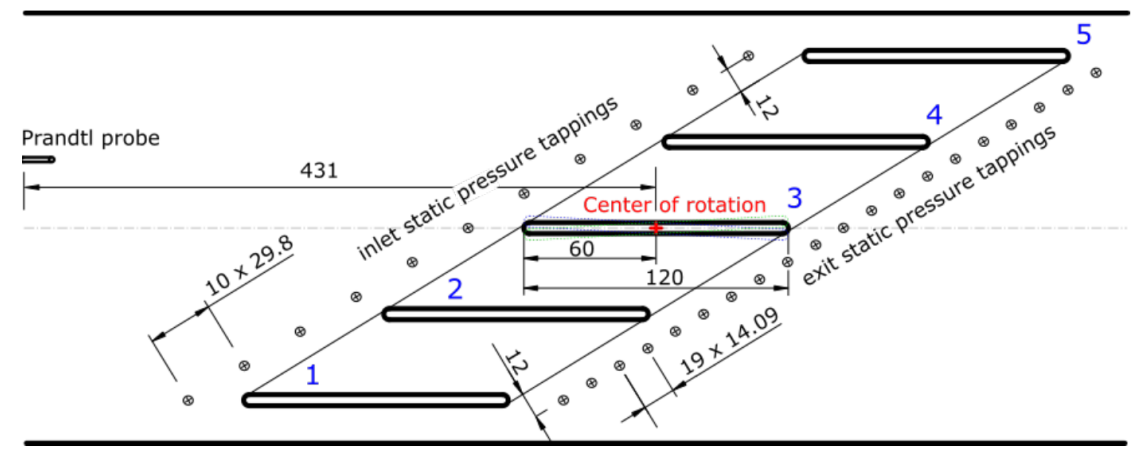

Figure 2: Scheme of the test section and measurement setup, dimensions are given in mm.

The outlet isentropic Mach number in the study was set as $M_{2, i s}=0.8$ and the middle blade setting as $\alpha \in\left\{-3^{\circ}, 0^{\circ},+3^{\circ}\right\}$. The reference Mach numbers $M_{o}$ corresponding to the outlet isentropic Mach number $M_{2, i s}=0.8$ for each blade setting are given as

$$
\left.M_{o}\right|_{\left(\alpha=-3^{\circ}\right)}=1.049,\left.\quad M_{o}\right|_{\left(\alpha=0^{\circ}\right)}=1.018,\left.\quad M_{o}\right|_{\left(\alpha=+3^{\circ}\right)}=0.996
$$




\subsection{Numerical computations}

Numerical simulations were carried out by means of the two-dimensional Favre-averaged NavierStokes equations for compressible gas. The thermophysical parameters of the gas were set to approximate the properties of air, i.e., the ideal gas equation of the state was used with the specific heat at constant pressure $C_{p}=1005 \mathrm{~J} / \mathrm{kg} / \mathrm{K}$. The dynamic viscosity $\mu=1.81 \times 10^{-5} \mathrm{~Pa} \cdot \mathrm{s}$ and Prandtl number $\operatorname{Pr}=0.72$ were considered constant. The turbulence effects were determined using the eddy viscosity approach within the RANS framework. The three-equation turbulence model with laminar-turbulent transition, $\gamma$-SST was used to close Navier-Stokes equations [8].

The inlet boundary conditions were set as follows: inlet total pressure $p_{1, t o t}=1 \times 10^{5} \mathrm{~Pa}$, inlet total temperature $T_{1, t o t}=288.15 \mathrm{~K}$, horizontal direction of velocity vector $\vec{u}$, kinetic turbulent energy intensity $T u=2 \%$ and viscosity ratio $\mu_{T} / \mu \approx 100$. Outlet boundary conditions were determined by pressure $p_{2}=65,602 \mathrm{~Pa}$, which corresponds to the outlet isentropic Mach number $M_{2, i s}=0.8$.

The numerical solution was obtained with a second-order finite-volume method using solver based on the OpenFOAM platform [9]. The solver uses approximate Riemann solvers for the evaluation of convective fluxes, combined with a central approximation of viscous terms. The steady-state solution was calculated with the pseudo-time stepping using a matrix-free LU-SGS method [10].

The computational mesh consists of 161,431 rectangular and triangular shape. A detail of section of the mesh near the middle blade is shown in Figure 3. The nearest cell center is in the distance from the wall about $y+\approx 1$ in average.

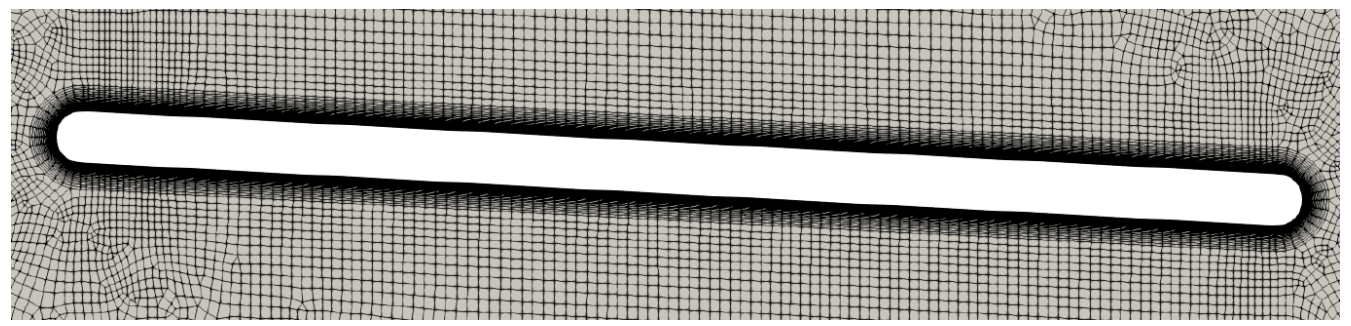

Figure 3: Detail of the mesh in the vicinity of the middle blade, $\alpha=+3^{\circ}$.

\subsection{Experimental measurements}

Throughout the experiments, the inlet total pressure was measured by a Prandtl probe positioned in the inlet of the channel (Figure 2). Inlet and outlet Mach numbers $M_{i}$ and $M_{o}$ were evaluated from the total pressure and average static pressures measured by the static pressure taps on the sidewalls upstream and downstream of the blade cascade. The positions of the 20 static pressure tappings downstream of the blade cascade corresponded to 20 probes used in CFD for evaluation of the reference isentropic Mach number $M_{o}$. Static pressure distributions on the blade surfaces used for evaluation of isentropic Mach number distributions were measured by 10 static pressure taps on one side of the blade. The distributions on the both sides of blades 2,3 and 4 were then obtained by placing the taps to different positions.

\section{Results}

The computed field of Mach number isolines in the vicinity of the cascade for incidence angles $\alpha=-3^{\circ}, 0^{\circ}$ and $+3^{\circ}$ is given in Figures 4-6. The series of shock waves are formed in the channel, profoundly affecting the character of developed flow field. One can see that with variation of incidence angle the inner and outer branch of exit shock wave of the blade 1 is not changed as well as flow field in the vicinity of lower and upper part of the blade 5. Thus, important changes of the positions and patterns of flow structures take place in the areas enclosing blades 2,3 and 4 . 


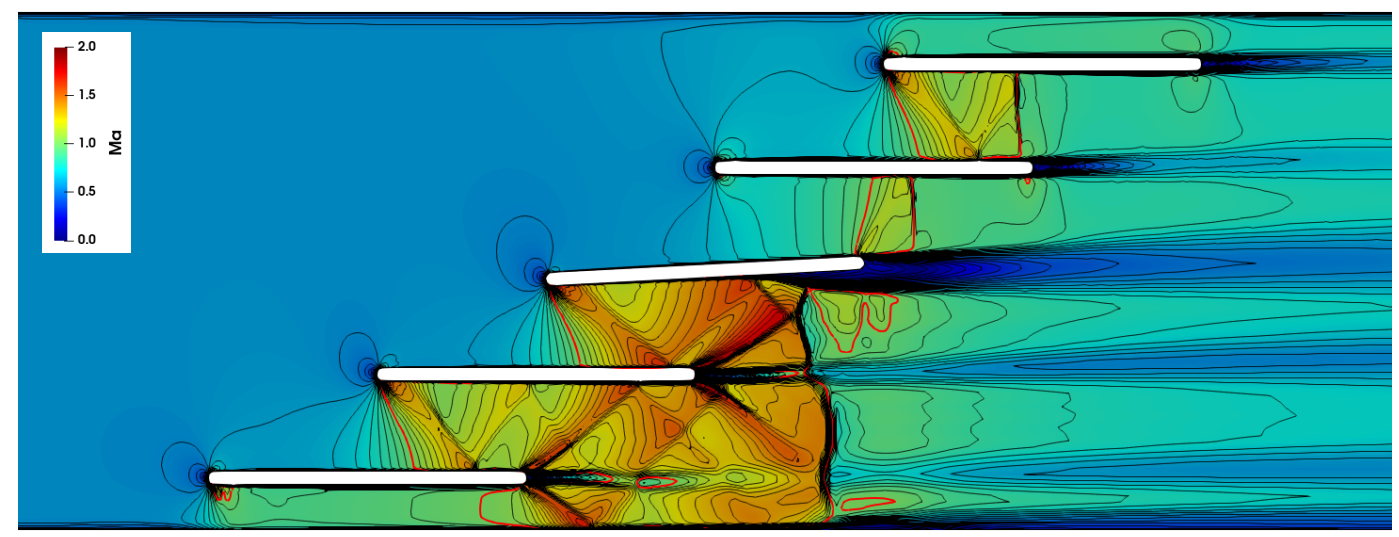

Figure 4: Distribution of the Mach number in the domain, $M_{2, i s}=0.8, \alpha=-3^{\circ}$.

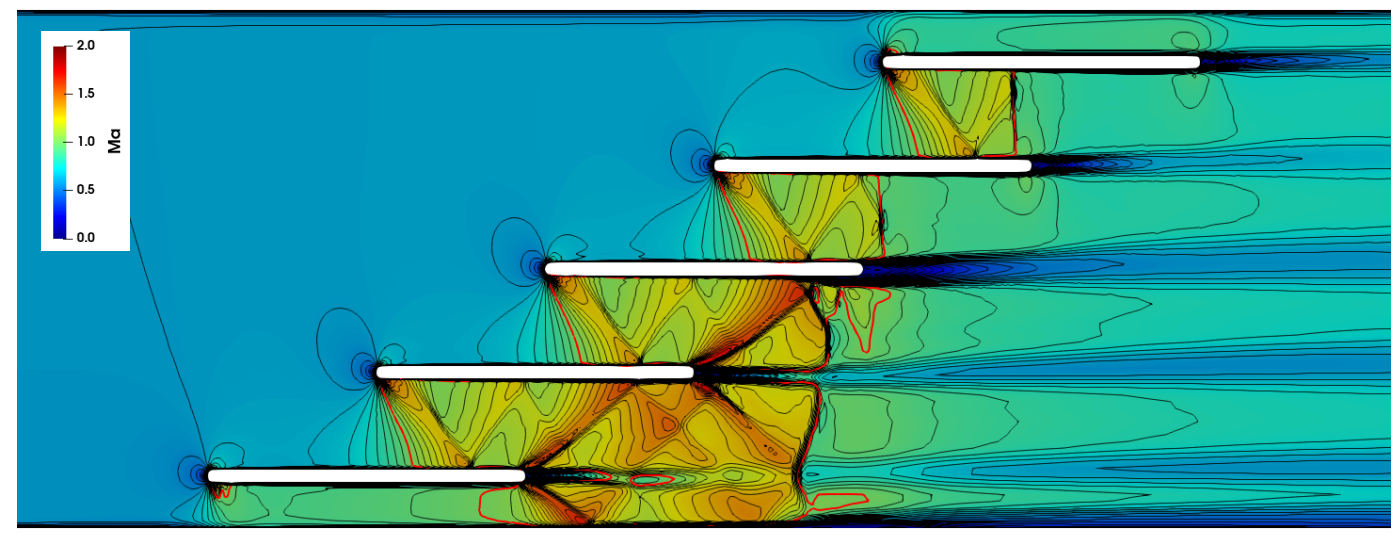

Figure 5: Distribution of the Mach number in the domain, $M_{2, i s}=0.8, \alpha=0^{\circ}$.

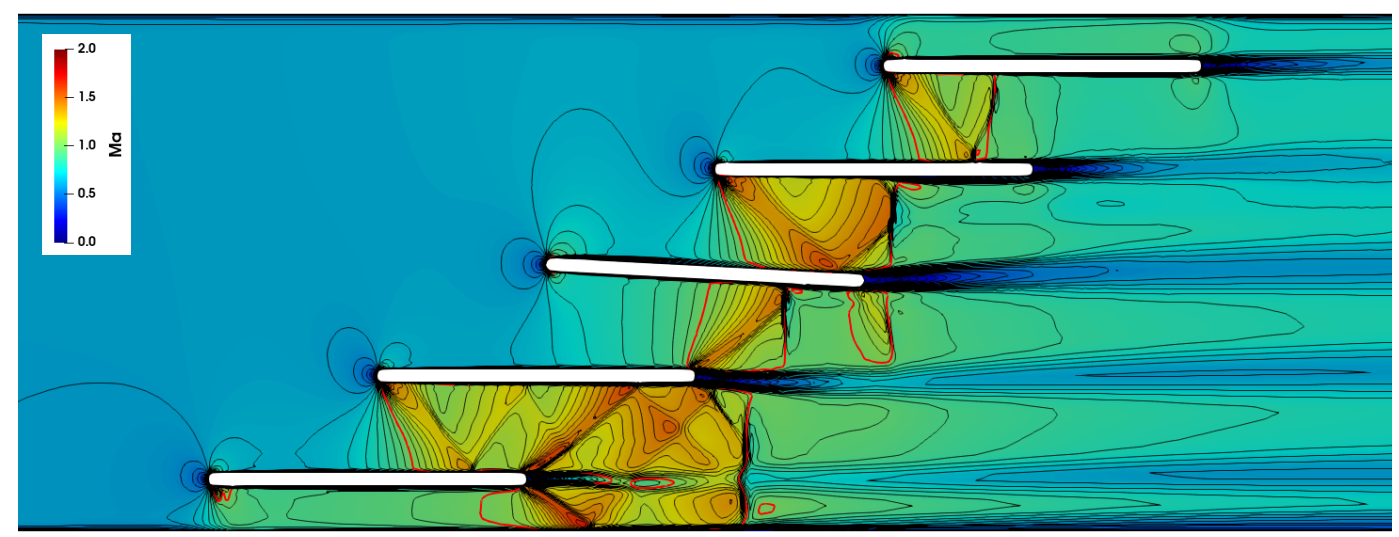

Figure 6: Distribution of the Mach number in the domain, $M_{2, i s}=0.8, \alpha=+3^{\circ}$.

For the negative inlet angle, $\alpha=-3^{\circ}$; see Figure 4 , the channel between blades 3 and 4 is acting as a convergent section of a nozzle and sonic speed is achieved at nearby the trailing edge of blade 3 and the position about $x / c=0.5$ on the lower side of blade 4 . The channel enclosed by blades 2 and 3 forms the divergent section of nozzle on the contrary. The sonic speed line spans between the leading edge of blade 3 and position about $x / c=0.65$ on the upper side of blade 2 . Just downstream of the sonic line a lip shock takes place on the lower surface of blade 3 at some distance from the leading edge. This shock wave is a result of interaction between expansion along the part of the surface where round leading edge joins straight part of the surface and the sonic line. This shock wave reflects from the upper surface of blade 2. Similar configuration just downstream the leading edge can be observed in the channel between blades 1 and 2 only at slightly lower velocities. Downstream of both blades 1 and 2 a typical system of exit shock waves takes place. However, 
accelerated supersonic flow downstream of interblade channels 12 and 23 can not be maintained at the given backpressure. Therefore a normal shock originates on the lower surface of blade 3 at about $x / c=0.75$ where it causes a massive flow separation. Typically, the separation point is located upstream of the normal shock resulting in a lambda shock configuration. In the region between the wake of blade 2 down to the wall there is series of shock reflections accompanied by weakened wake of blade 1 containing subsonic spots, finally resulting in normal shock wave which spans from channel wall and tie up with the lambda shock.

In the case of zero inlet angle, $\alpha=0^{\circ}$; see Figure 5, the flow field from bottom wall up to the horizontal axis intersecting middle blade can be regarded as the limit case of the negative inlet angle setting and; correspondingly, the flow field from the horizontal axis up to the top wall can be viewed as the limit case of positive inlet angle setting.

The positive angle setting, $\alpha=+3^{\circ}$; see Figure 6 , results in the divergent section of nozzle between blades 3 and 4 . The sonic line spans form the lower side of leading edge of the blade 4 down to the upper side of blade 3 at position about $x / c=0.6$. Supersonic expansion with presence of shock reflections then follows downstream resulting in the lambda shock developed due to the convergent shape of the near wake of blade 3 and the boundary layer of blade 4 . The channel between blades 2 and 3 is convergent up to the trailing edge of blade 2 where the channel diverges and flow accelerates to supersonic velocity resulting in origin of exit shock wave on the upper surface of blade 2. Downstream the sonic line supersonic region (red square in Figure 6) containing the inner branch of exit shock wave of blade 2 is formed. Due to relatively high backpressure, a normal shocks form downstream of blades 1,2 and 3. Causing wake thickening and local flow separations on lower surfaces of blades 2 and 3. Another supersonic region is formed downstream the trailing edge of the blade 3; similarly, due to the convergent-divergent-convergent shape of wake of blade 2 and near wake of blade 3 . The fact that flow accelerates to supersonic velocities even in the straight interblade channels suggests that the inlet flow angle just upstream of the blade cascade is shifted towards negative values, i.e. velocity vectors direct downwards.

Figure 7 shows the distribution of the isentropic Mach number along the inlet and outlet probe lines for angles $\alpha=-3^{\circ}$ and $+3^{\circ}$. The experimental data taken in 11 evenly distributed pressure probes along the inlet line and 20 probes along the outlet line are also depicted. The coordinate $l$ is measured from the lower left point of the line (see Figure 2). The isentropic Mach number is calculated using the following formula

$$
M_{i s}=\sqrt{\frac{2}{\gamma-1}\left[\left(p / p_{1, t o t}\right)^{\frac{1-\gamma}{\gamma}}-1\right]},
$$

where $p$ is the pressure evaluated from inlet resp. outlet readings.
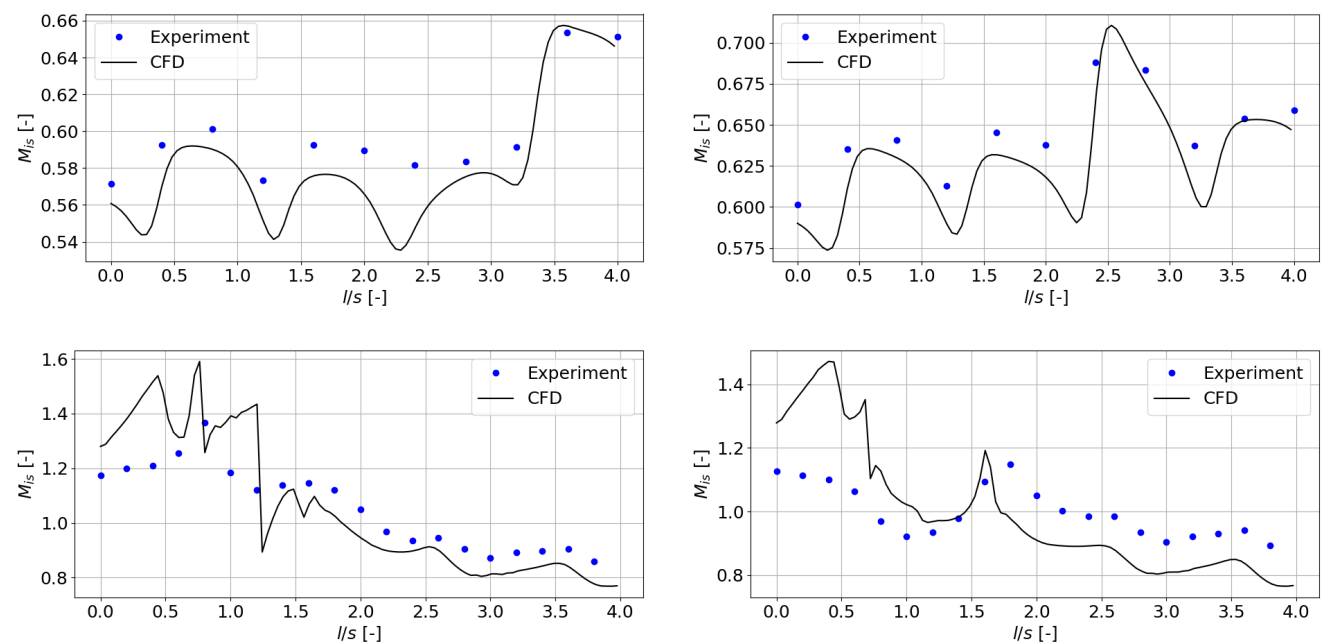

Figure 7: Isentropic Mach number distribution along the inlet (upper row) and outlet (lower row) probe line. Left pane $\alpha=-3^{\circ}$, right pane $\alpha=+3^{\circ}$. 
The distribution of the isentropic Mach number obtained by means of CFD corresponds with values calculated from experimentally measured pressures quite acceptably in the inlet part considering the range of plotted values. Distributions suggest that the aperiodicity is slightly less pronounced in case of experiment. The outlet isentropic Mach number values agree well with CFD from blade 2 up. The inconsistency in the part $0<l / s<1.2$ on the outlet probe line is discussed furthermore.

In the case of inlet distribution at the angle $\alpha=-3^{\circ}$ there is apparent slow oscillatory decrease in the values form blade 1 up to blade 4 . The reduced flow rate in the channel between blades 2 and 3 and accelerated flow in the convergent shape of the channel between blades 3 and 4 deflecting the flow direction up results in the isentropic Mach number rise upstream the channel between blades 4 and 5 . On the contrary, in the case of $\alpha=+3^{\circ}$ the values of isentropic Mach number are increasing from bottom to top. The convergent part is moved down, between blades 2 and 3 , whilst the geometrical configuration allows to reach higher flow rate between blades 2 and 3 . This affects the flow direction in a way that peak of isentropic Mach number is shifted to the vicinity of blades 3 and 4 where higher flow rate is also achieved in comparison with the configuration of $\alpha=-3^{\circ}$.

The outlet isentropic Mach number distribution has similar course for both settings $\alpha=+3^{\circ}$ and $-3^{\circ}$. The values are decreasing from the bottom to top as supersonic expansion in the vicinity of trailing edges occur in the lower part of the channel.
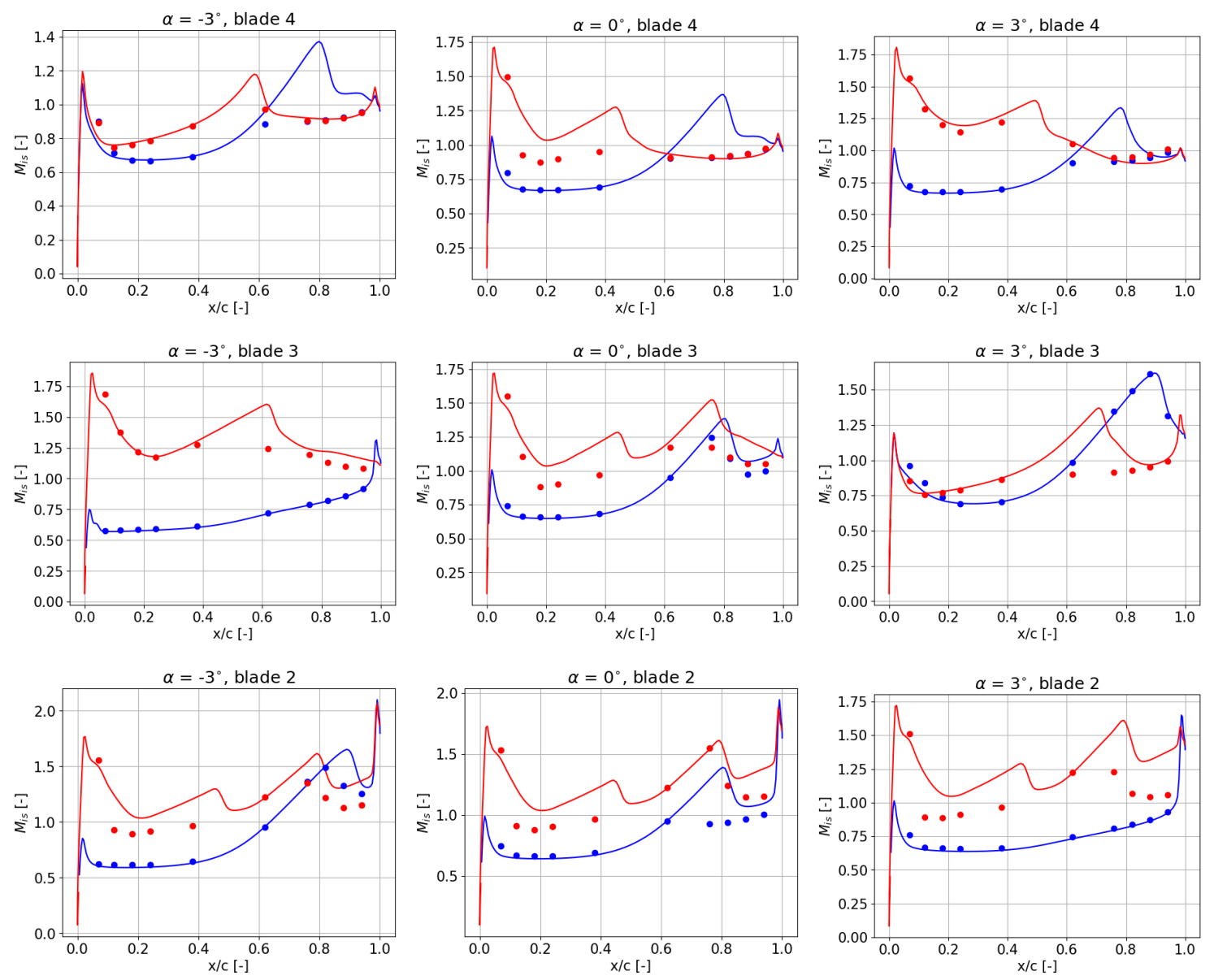

Figure 8: Isentropic Mach number distribution along blades 2, 3 and 4 at middle blade settings $\alpha=-3^{\circ}, 0^{\circ}$ and $+3^{\circ}$. Solid lines - CFD, symbols - Experimental, red - lower surface, blue - upper surface.

The discrepancies between measured and computed values, apart from the fact that the simulation is $2 \mathrm{D}$ and experiment inevitably $3 \mathrm{D}$, can be partially explained by the presence of shock 
waves disturbing the flow field and also by the fact that the pressure taps were mounted on the sidewalls of the channel, and thus possibly influenced by boundary layers.

The comparison of experimental and CFD distribution of the isentropic Mach number along blades 2, 3 and 4 for various values of inlet angle $\alpha$ can be seen in Figure 8 . In the case of blade 4 (first row), the experimentally obtained values of isentropic Mach number on both lower and upper blade surface match quite well with CFD results for $0<x / c<0.6$. From $x / c=0.6$ downstream the upper surface experimental values are lowered against CFD values. The possible explanation is the presence of supersonic region between blades 4 and 5 in numerically obtained flow field while in the course of experiments the range of supersonic region was possibly different due to a complex development of sidewall boundary layers. The middle row on Figure 8 shows distribution along blade 3 at different settings. The experimental data agree well with CFD results, especially on the upper surface of the blades. Measured isentropic Mach number values on the lower surfaces of the blades start to differ from the CFD results from $x / c=0.6$ downstream in the case of $\alpha=+3^{\circ}$ due to the presence of aforementioned supersonic region between blades 2 and 3 . The distribution along blade 2 exhibits the same qualitative behavior as in the case of blade 2 and 3 . In overall, the experimental data differ from CFD results in the locations where the impact of shockwave is present. Such flow configuration is typically subject to a relatively strong influence of the sidewall boundary layer thickness development which change effective shape of the interblade channels.
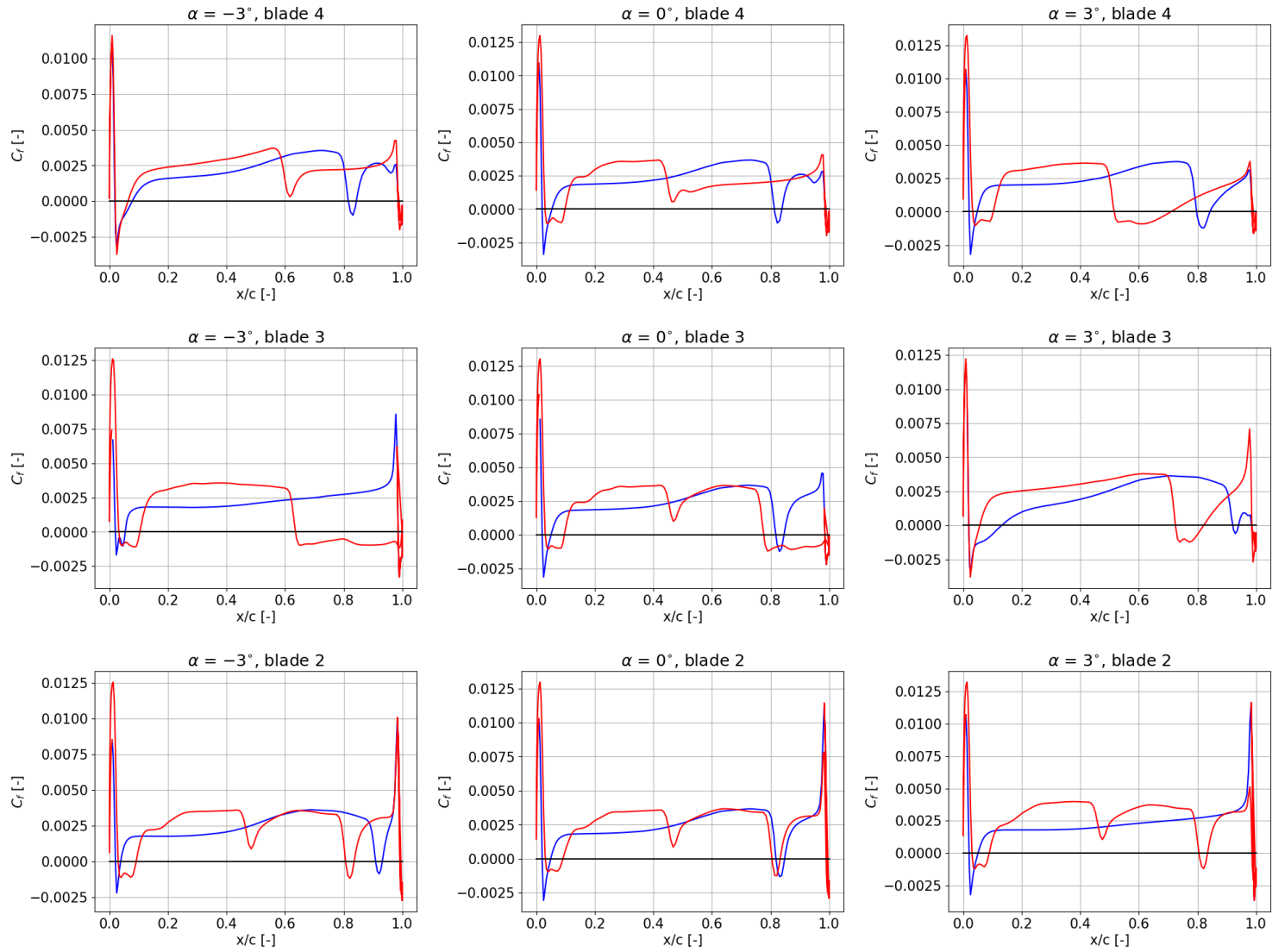

Figure 9: Distribution of the skin friction coefficient along blades 2, 3 and 4 at middle blade settings $\alpha=-3^{\circ}, 0^{\circ}$ and $+3^{\circ}$, red - lower surface, blue - upper surface.

The positions of shock waves and separation bubbles on blades 2, 3 and 4 can be also detected from the skin friction coefficient, $C_{f}=-2 \tau_{w} /\left(\rho_{2, i s} U_{2, i s}^{2}\right)$, distribution in the Figure 9 . Here $\tau_{w}$ is the wall shear stress and the reference quantities $\rho_{2, i s}$ and $U_{2, i s}^{2}$ are the outlet isentropic density and the velocity magnitude, respectively. The separation bubbles can be identified by negative $C_{f}$ and the positions of shock waves rapid drop in the $C_{f}$ value. 


\section{Conclusions}

The results of experimental investigation of the effect of incidence angle offset in a two-dimensional section of a flat linear blade cascade in high-speed wind tunnel were compared with CFD results obtained by means of finite-volume, in-house code developed on the top of OpenFOAM framework. Predictions were concentrated on the comparison of data obtained at the inlet and outlet of the cascade and also the physical quantities on the surface of the blades. The experimental and numerical results match quite well except where the shock wave - boundary layer interaction occurs. Differences were attributed mainly to the fact that simulations were two dimensional and thus could not reflect influence of sidewall boundary layers development. Three dimensional simulations are planned in the future as well as the investigation of non-stationary setup of the oscillating middle-blade.

\section{Acknowledgments}

The work was supported by the Czech Science Foundation (GACR) under Grant no. Grant No. 20-11537S, by the Institute of Thermomechanics of the Czech Academy of Sciences with institutional support RVO: 61388998, and by the Student Grant Scheme at the Czech Technical University in Prague through project no. SGS-OHK2-004/19.

\section{References}

[1] Bölcs, A.: A test facility for the investigation of steady and unsteady transonic flows in annular cascades in Turbo Expo: Power for Land, Sea, and Air 79511 (1983), V001T01A014.

[2] Rottmeier, F.: Experimental investigation of a vibrating axial turbine cascade in presence of upstream generated aerodynamic gusts, 164. http://infoscience.epfl.ch/record/ 103723 (2003).

[3] Vogt, D. M. \& Fransson, T. H.: A new turbine cascade for aeromechanical testing in Proceedings of the 16th Symposium on Measuring Techniques in Transonic and Supersonic Flow in Cascades and Turbomachines (2002).

[4] Vogt, D. M. \& Fransson, T. H.: Experimental Investigation of Mode Shape Sensitivity of an Oscillating Low-Pressure Turbine Cascade at Design and Off-Design Conditions. Journal of Engineering for Gas Turbines and Power 129, 530-541. ISSN: 0742-4795. eprint: https: / / asmedigitalcollection . asme . org / gasturbinespower / article-pdf / 129 / 2/530 / 5587129/530\_1.pdf. https://doi.org/10.1115/1.2436567 (Aug. 2006).

[5] Fürst, J., Lasota, M., Musil, J. \& Pech, J.: Numerical Investigation of Aeroelastic Flutter in Two-Dimensional Cascade of Compressor Blades. MATEC Web Conf. 328, 02020. https: //doi.org/10.1051/matecconf/202032802020 (2020).

[6] Fürst, J. et al.: Effects of a Single Blade Incidence Angle Offset on Adjacent Blades in a Linear Cascade. Processes 9. ISSN: 2227-9717. https : //www . mdpi . com/2227-9717/9/11/1974 (2021).

[7] Lepicovsky, J., Šidlof, P., Šimurda, D., Štěpán, M. \& Luxa, M.: New test facility for forced blade flutter research in AIP Conference Proceedings 2323 (2021), 030001.

[8] Menter, F. R., Smirnov, P. E., Liu, T. \& Avancha, R.: A One-Equation Local CorrelationBased Transition Model. Flow, Turbulence and Combustion 95, 583-619. ISSN: 1573-1987. https://doi.org/10.1007/s10494-015-9622-4 (Dec. 2015).

[9] Weller, H. G., Tabor, G., Jasak, H. \& Fureby, C.: A tensorial approach to computational continuum mechanics using object-oriented techniques. Computers in physics 12, 620-631 (1998).

[10] Jiří, F.: Development of a coupled matrix-free LU-SGS solver for turbulent compressible flows. Computers \&6 Fluids 172, 332-339. ISSN: 0045-7930. https://www. sciencedirect. com/science/article/pii/S0045793018302160 (2018). 